\title{
The predictors of interpregnancy change in body mass index
}

\begin{abstract}
Introduction: Pregnancy is a time when weight increases as part of a physiological process to aid fetal growth. However, when excess weight is gained during pregnancy, and retained thereafter, the risk of obesity in the future increases. Increasing BMI between pregnancies and maternal obesity are associated with several adverse pregnancy outcomes. Although the risks of increasing BMI on future pregnancies is well defined, the predictors of this weight gain are not. This study aimed to investigate the predictors of interpregnancy BMI change.
\end{abstract}

Materials and methods: This study was conducted in one of Europe's largest maternity hospitals delivering approximately 8000 infants per annum. Women's sociodemographic and clinical data were self-reported at the first antenatal visit and computerised to an electronic recording system by trained midwives. Weight and height were measured at the first antenatal visits of both pregnancies, and body mass index was calculated. Data was extracted and analysed for women who delivered their first and second singleton infants between 2009-2018.

Results: A total of 12,056 women delivered their first and second baby over the 10 -year period. The mean interval between pregnancies was 32.3months (SD15.9) and the median BMI change was 0.6units (IQR1.3). From the first to the second pregnancy the rate of obesity increased from $11.6 \%$ to $16.0 \%$. Between pregnancies $46.1 \%$ of women maintained their BMI ( -1 to +1 units), $13.3 \%$ lost $>1$ BMI unit(s), whereas $15.5 \%$ gained $1-2$ unit(s), $9.9 \%$ gained $2-3$ units and $12.0 \%$ gained $>3$ units. Overall, $5.8 \%$ became obese by the second pregnancy. On multinomial regression analysis, having a pregnancy interval of $>3 y$ ears $(\mathrm{aOR} 2.1,95 \% \mathrm{CI} 1.9-2.5$, $\mathrm{p}$ $<0.001$ ), artificial feeding after the first pregnancy (aOR1.8, 1.5-2.0, p < 0.001), postnatal depression after the first pregnancy $(\mathrm{aOR} 1.6,1.3-2.1, \mathrm{p}<0.001)$ and taking prescribed anxiolytics or antidepressants $(\mathrm{aOR} 1.6,1.1-2.5$, $\mathrm{p}=0.013)$ were predictors of gaining $>3$ BMI units between pregnancies after adjusting for maternal occupation and age. The predictors of becoming obese in the second pregnancy also included a pregnancy interval of $>3$ years (aOR 1.5, 1.2-1.8, p < 0.001), artificial feeding after the first pregnancy (aOR2.1, 1.8-2.6, p<0.001), postnatal depression after the first pregnancy $(\mathrm{aOR} 1.7,1.2-2.3, \mathrm{p}=0.001)$ and taking prescribed anxiolytics or antidepressants $(\mathrm{aOR} 1.8,1.1-3.1, \mathrm{p}=0.016)$ following the same adjustments.

Conclusion: Longer pregnancy interval, not breastfeeding and psychological health disorders are predictors of BMI increase between pregnancies and becoming obese in the second pregnancy. Interventions provided following women's first delivery should aim to promote breastfeeding, manage weight and improve mental health.

\section{Conflict of Interest}

There is no conflict of interest 